\title{
Analysis of Ice Structure Formed in Frozen Agar Gel
}

\author{
Osato MiYawaki ${ }^{1}$, Tomoyuki FusII ${ }^{2}$ and Yoko ShimiYa ${ }^{3}$ \\ ${ }^{1}$ Department of Applied Biological Chemistry, The University of Tokyo, 1-1-1 Yayoi, Bunkyo-ku, Tokyo 113-8657, Japan \\ ${ }^{2}$ Department of Food Science, Niigata University of Pharmacy and Applied Life Sciences, Niitsu, Niigata 956-8603, Japan \\ ${ }^{3}$ Department of Food and Health Sciences, Jissen Women's University, 4-1-1 Osakaue, Hino, Tokyo 191-8510, Japan
}

Received April 24, 2004; Accepted August 26, 2004

\begin{abstract}
Ice crystal structure was analyzed for frozen agar gel under unidirectional freezing in various operating conditions to elucidate the mechanism of the process of ice crystal growth. A linear relationship was obtained between the mean ice crystal size and the inverse of the moving speed of the freezing front. The slope of the correlation was in the same order as the diffusion coefficient of water, suggesting the important role of the molecular diffusion in this growth process. No meaningful effect of the agar concentration was observed on the ice structure showing that the agar matrix structure does not affect the ice structure in an agar concentration ranging from 1.5 to $5 \%$.
\end{abstract}

Keywords: unidirectional freezing, ice crystal structure, dendrite, diffusion of water

In frozen food, ice structure is very important to control its quality (Trgo et al., 1999; Russel et al., 1999; Regand and Goff, 2002, 2003). The ice structure also strongly affects the behavior of freeze-related operations such as freeze drying, freeze concentration, freeze texturization, and freeze smashing (Miyawaki, 2001).

Various methods have been reported in the literature to observe the ice structure formed in frozen food. Bevilacqua et al. (1979), Miyawaki et al. (1992), and Chen and Pan (1997) chemically fixed the frozen food structure and measured the holes left by the ice structure after thawing under a microscope. Bomben and King (1982), and Ngapo et al. (1999) used cryo-scanning electron microscopy. Freeze drying technique has been applied by several groups. Woinet et al. (1998b) and Chevalier et al (2000) analyzed the hole left after freeze drying as a trace of the ice structure by microscope and Bae et al. (1993) applied mercury porosimetry to analyze the hole size distribution. For the measurement of ice crystals in situ, Evans et al. (1996) used a confocal laser scanning microscope and Kerr et al. (1998) employed magnetic resonance imaging. Bolliger et al. (1999) applied near infrared spectroscopy to measure structure parameters of frozen sorbet.

To analyze the mechanism of ice crystal growth, control of the heat flow in the freezing process is indispensable. For this purpose, unidirectional freezing has frequently been employed. Bevilacqua et al. (1979) analyzed the diameter of the intracellular dendrites and extracellular ice crystals formed in unidirectionally frozen beef. Bomben and King (1982) observed the ice crystal structure in frozen apple tissue and showed that the dendritic spacing of the ice was proportional to the inverse square root of the cooling rate by applying the theoretical work based on dendritic solidification of aluminum-copper alloys by Rohatgi and Adams

E-mail: aosato@mail.ecc.u-tokyo.ac.jp (1967ab). Miyawaki et al. (1992) and Bae et al. (1993) applied the dimensional analysis approach to analyze ice crystal structures formed in protein and agar gels and pointed out the important role of the molecular diffusion in the ice crystal growth process. Woinet et al. (1998b) combined the theoretical result by Rohatgi and Adams (1967ab) with the Neumann model for heat transfer with phase transition (Woinet et al., 1998a) to evaluate the effect of the initial temperature on the ice structure formed in frozen gelatin gels. Chevalier et al. (2000) analyzed the ice structure formed in cylindrical gelatin gels unidirectionally frozen in the radius direction.

In the present paper, ice crystal structure was analyzed for the frozen agar gel under unidirectional freezing in various operating conditions to elucidate the mechanism of the ice crystal growth process. Results were compared with ice structures formed in different systems in the literature to evaluate the contribution of the molecular diffusion in this process. Ice structures were also compared among different agar concentrations to elucidate the effect of agar matrix structure on them.

\section{Materials and Methods}

Materials and sample preparation Agar powder (Nacalai Tesque Co., Tokyo) was dissolved in hot deionized water and $1.5,3.0$, or $5.0 \mathrm{wt} \%$ agar solution thus prepared was immediately poured into a sample vessel to form a gel before freezing.

Apparatus and method for unidirectional freezing The apparatus for the unidirectional freezing is shown in Fig.1. The sample vessel was composed of a cylindrical acrylic resin ( $2 \mathrm{~mm}$ thick) $26 \mathrm{~mm}$ in inner diameter and an aluminum cooling plate $20 \mathrm{~mm}$ thick at the bottom mounted on a thermo-module. Temperature of the cooling plate was controlled by a thermo-module controller (NetsuDenshi, Tokyo, MT-602) in the range from room temperature to $-40^{\circ} \mathrm{C}$. The sample vessel was thermally insulat- 


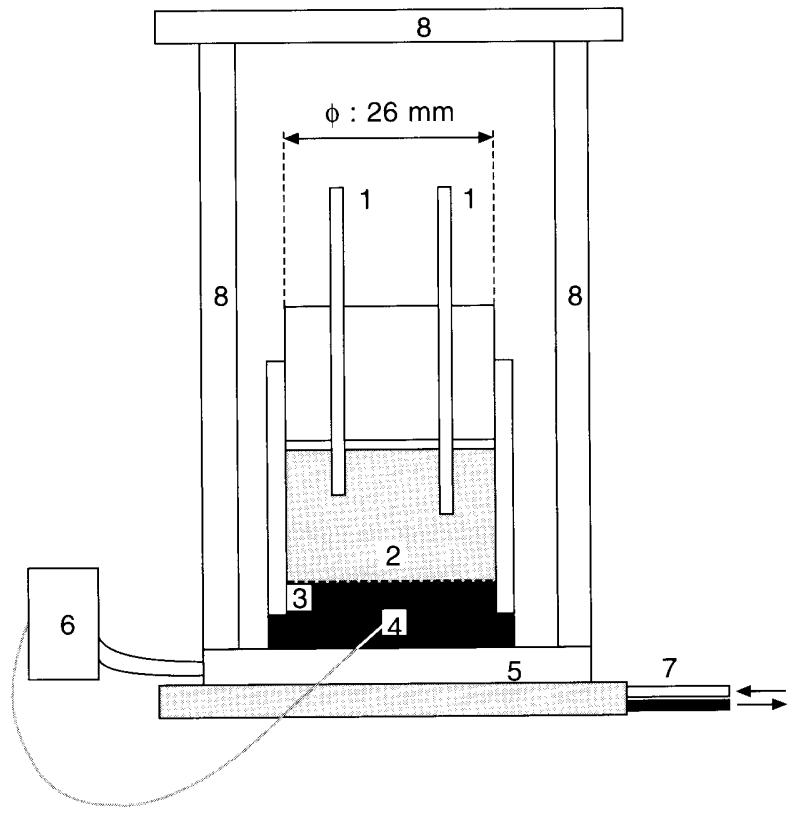

Fig. 1. Apparatus for unidirectional freezing. (1, thermocouple; 2 , sample gel; 3 , cooling plate; 4 , sensor; 5 , thermo-module; 6 , thermomodule controller; 7 , cooling water; 8 , insulator).

ed by air surrounded by a cylindrical acrylic resin. This insulation was enough to guarantee the unidirectional freezing because the plane ice front was always observed perpendicular to the direction of heat flow. The sample vessel was equipped with two copper-constantan thermocouples placed just below and above the sampling point for the ice structure analysis to accurately measure the moving speed of the freezing front. The cooling plate of the sample vessel filled with agar solution was initially kept at $5^{\circ} \mathrm{C}$ to form a gel. Then, the temperature of the cooling plate was stepped down to a desired temperature to start the unidirectional freezing.

Measurement of the moving speed of the freezing front From the temperature change, the time for the passage of freezing front in the frozen agar gel was measured at 2 different positions, as explained above. The moving speed of the freezing front, $U$, was calculated from the difference of the passage time of the freezing front and the distance between the two thermocouples, typically 1.5 to $3 \mathrm{~mm}$.

Measurement of ice structure size After the completion of freezing, the frozen agar gel sample was taken out and transferred to a freeze-drier (Eyela, Tokyo, FD550) quickly without thawing. The freeze-dried gel was cut into slices $2 \sim 3 \mathrm{~mm}$ thick by a thin stainless razor in the section perpendicular to the direction of heat flow at the middle position between the two thermocouples. The porous structure of the sample gel was measured and recorded by a video-microscope (Keyence, Osaka, VH5910). The image data were analyzed by software (NIH image, NIH, Bethesda, MD).

\section{Results and Discussion}

Ice crystal structure formed in frozen agar gel by unidirectional freezing Figure 2 shows temperature changes measured at various positions in the freezing process of an agar gel. Temperature measured on the cooling plate and that at $0.8 \mathrm{~mm}$ from the cooling plate clearly showed supercooling, which is empirically known to cease simultaneously in the range where supercooling occurs (Miyawaki et al., 1989). At $4.0 \mathrm{~mm}$ and $7.4 \mathrm{~mm}$ apart from the cooling plate, no supercooling was observed. As the freezing point depression was negligible, in the present case, the freezing front was considered to pass through the sensor location when its temperature reached $0^{\circ} \mathrm{C}$.



Fig. 2. Temperature change in the freezing process of agar gel measured at various positions apart from the cooling plate. $(-$, cooling plate; -----, $0.8 \mathrm{~mm} ;---, 4.0 \mathrm{~mm} ;-\cdot-, 7.4 \mathrm{~mm}$ apart from the cooling plate)

Figure 3 shows the ice structure formed in 3\% agar gel observed in the plane parallel to the heat flow. At the lower part close to the cooling plate $(3 \mathrm{~mm}$ above the cooling plate), a zone with very fine ice structure was observed because of the supercooling. From this supercooling zone, a dendrite structure grew parallel to the direction of heat flow. At the higher part ( $7 \mathrm{~mm}$ above the cooling plate), the ice structure was totally composed of this dendrite structure. Figure 4 shows the dendrite structures observed in the plane perpendicular to the heat flow. It is evident that ice crystal size increased with an increase in the distance from the cooling plate.

Analysis of ice structure size Size distribution of the ice structure was analyzed for the minor (smallest) and major (largest) axes. Number of samples analyzed was from 400 to 600 in each case. Typical results are shown in Fig. 5 . The mean major axis was about two fold larger than the mean minor axis in this case. Similar analyses were carried out for agar gels with concentration varied from 1.5 to $5 \%$ and frozen at various operating conditions represented by the moving speed of the freezing front $(U)$. Results are listed in Table 1. In this table, correction value means a correction for drying shrinkage of the sample, which was determined by the ratio of the sample diameter before and after freeze drying. From the mean minor axis $\left(d_{1}\right)$ and the 


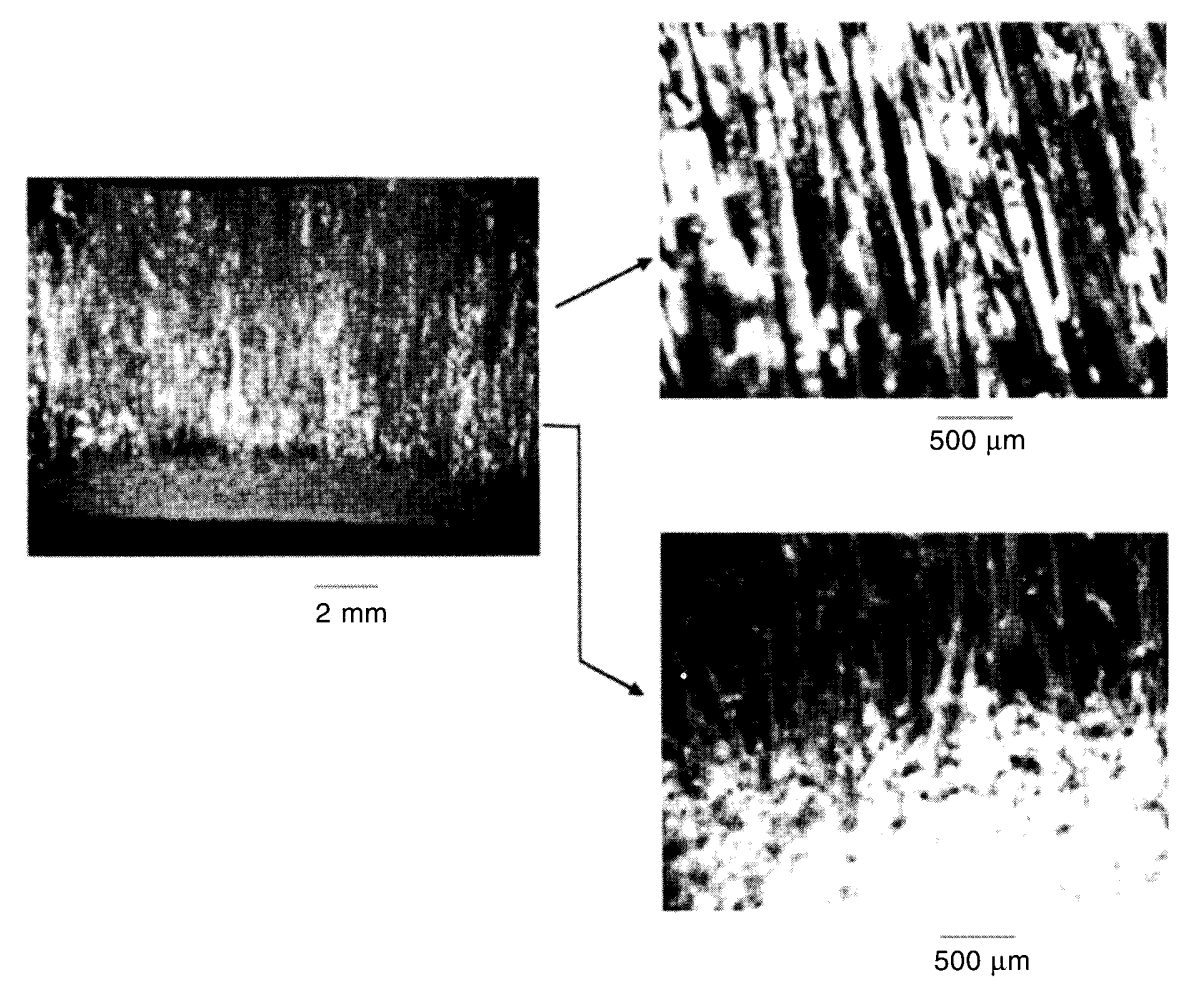

Fig. 3. Ice structure formed in frozen and freeze-dried $3 \%$ agar gel in the section parallel to the direction of heat flow.

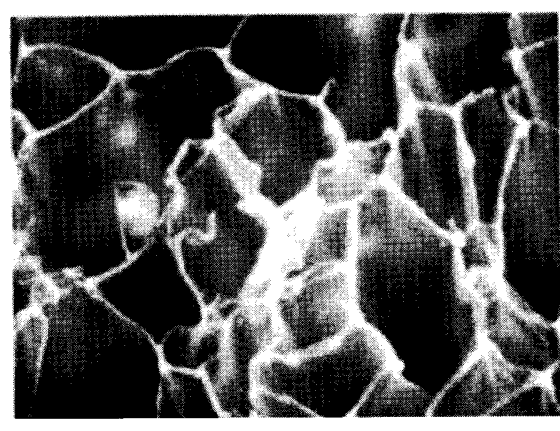

$\overline{200 \mu \mathrm{m}}$

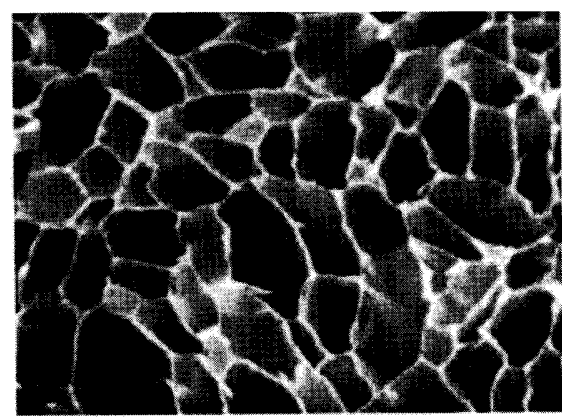

$\overline{200 \mu \mathrm{m}}$

B

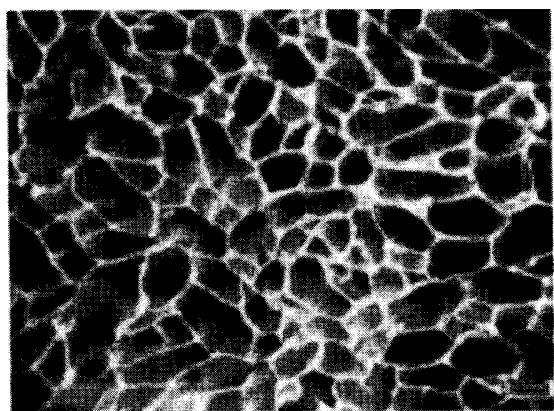

$\overline{200 \mu \mathrm{m}}$

C

Fig. 4. Ice structure formed in frozen and freeze-dried $3 \%$ agar gel in the section perpendicular to the direction of heat flow at $12 \mathrm{~mm}(\mathbf{A})$, $8 \mathrm{~mm}(\mathbf{B})$, and $5 \mathrm{~mm}(\mathbf{C})$ apart from the cooling plate.

mean major axis $\left(d_{2}\right)$, the geometric axis $\left(d_{\mathrm{P}}=d_{1} \times d_{2}\right)^{1 / 2}$ was calculated and plotted against the inverse of the moving speed of freezing front, $1 / U$, in Fig. 6. Although data seem a little scattered, linear relationships between $d_{P}$ and $1 / U$ were statistically observed for ice crystals formed in agar gels with concentration varied as follows:

$$
d_{\mathrm{P}}=\alpha / U+\beta
$$

where $\alpha$ and $\beta$ are experimental constant. No systematic effect of agar concentration was observed on $\alpha$ or $\beta$ suggesting there was no effect of gel structure on the ice structure in this concentration range of agar.

In our previous work (Miyawaki et al., 1992; Bae et al., 1993), we applied a dimensional analysis approach to obtain the relationship between $d_{P}$ and $1 / U$ as follows:

$$
d_{\mathrm{P}} U / D_{\mathrm{W}} \sim 1
$$

where $D_{\mathrm{W}}$ is an effective molecular diffusion coefficient involving water and solutes in the growth process of an ice crystal. From the comparison of Eqs. (1) and (2), $\alpha$ in Eq. (1) would correspond to $D_{\mathrm{W}}$ in Eq. (2). In Table 2, $\alpha$ or $U d_{\mathrm{P}}$ in the literature are compared. These are distributed relatively within a narrow range and not much different from the self diffusion coefficient of water $\left(1.0 \times 10^{-9} \mathrm{~m}^{2} / \mathrm{s}\right)$. This strongly suggests that the contribution of the molecular diffusion is very important in the mechanism of the ice crystal growth process and the slope in Fig. $6, \alpha$, seems to represent this diffusion process.

Bevilacqua et al. (1979) analyzed the ice crystal structure in frozen beef by applying the theory of Tiller and Rutter (1956) on binary alloy to obtain the following result for ice crystal size.

$$
d_{\mathrm{P}}=\alpha^{\prime} / U G+\beta^{\prime}
$$



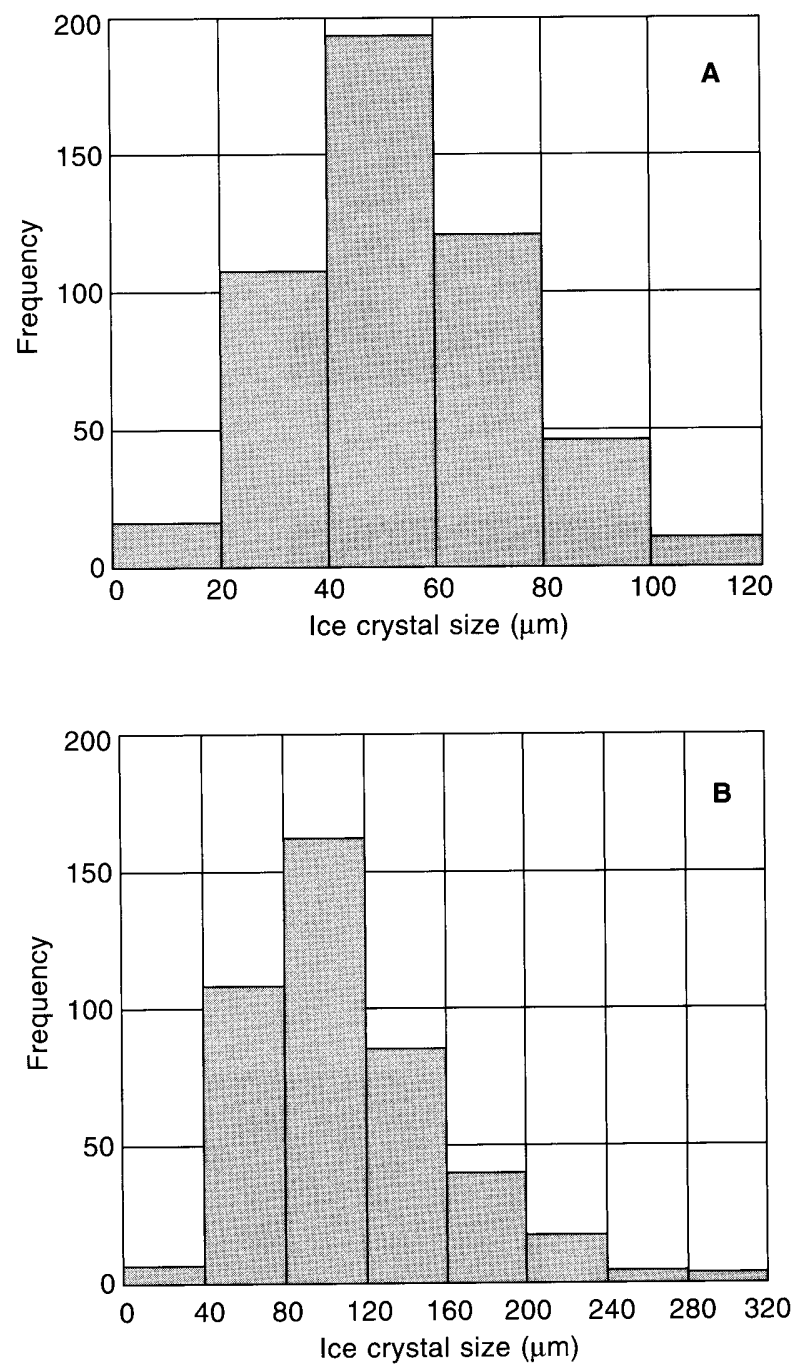

Fig. 5. Minor (A) and major (B) axis distribution of ice crystals formed in frozen and freeze-dried $1.5 \%$ agar gel in the section perpendicular to the direction of heat flow.

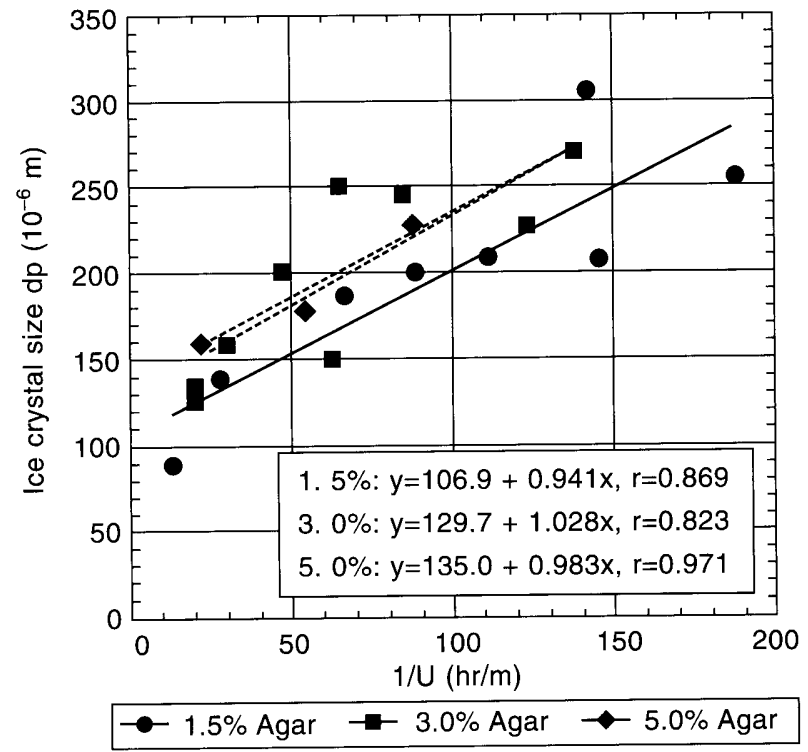

Fig. 6 Correlation between mean ice crystal size $\left(d_{\mathrm{p}}\right)$ formed in frozen agar gel and moving speed of the freezing front $(U)$. where $G$ is the temperature gradient at the freezing front.

Bomben and King (1982) applied the theory on the dendritic solidification of alloy by Rohatgi and Adams (1967ab) to analyze the dendritic spacing of the ice crystal $(L)$ formed in frozen apple tissue as follows:

$$
L \propto(U G)^{-1 / 2}
$$

As the temperature gradient $G$ is proportional to $U$ (Bomben and King, 1982), Eq. (3) will be rewritten as:

$$
d_{\mathrm{P}}=\alpha^{\prime} / U^{2}+\beta^{\prime}
$$

When the dendrite spacing is considered to be similar to $d_{\mathrm{P}}$, Eq. (4) will be:

$$
d_{\mathrm{P}} \propto 1 / U
$$

Therefore, Eq. (4) can be considered similar to Eqs. (1) or (2) and only Eq. (3) is different from the others. Equation (3), however, have been applied to alloy crystals ranging from 42 to $60 \mu \mathrm{m}$ (Tiller and Rutter, 1956) and to ice crystals in frozen beef ranging from 28 to $50 \mu \mathrm{m}$ (Bevilacqua et al., 1979). This narrow applicable range suggests the limit of this model. On the contrary, Eqs. (1), (2), and (4) have been applied to dendrite spacing formed in electrolyte solutions ranging from 50 to $180 \mu \mathrm{m}$ (Rohatgi and Adams, 1967a), dendrite spacing in frozen apple tissue ranging from 5 to $300 \mu \mathrm{m}$ (Bomben and King, 1982), ice crystals in frozen protein gels ranging from 5 to $500 \mu \mathrm{m}$ (Miyawaki et al., 1992), ice crystals in frozen agar gels ranging from 30 to $120 \mu \mathrm{m}$ (Bae et al., 1993), and ice crystals in agar gels ranging from 20 to $300 \mu \mathrm{m}$ in the present case. This wide applicability in size shows the effectiveness of this model.

Apart from these models, Chevalier et al. (2000) applied the following power law model (Reid, 1980) to analyze the ice structure formed in cylindrical gelatin gels frozen radially:

$$
d_{\mathrm{P}}=a U^{\mathrm{b}}
$$

They reported $b=-0.45$ as the best-fit. This large difference in the exponent $b$, as compared with other equations suggests that it reflects the difference between unidirectional freezing and radial freezing.

It is well known that the time for passage of the zone of maximum crystallization $\left(0 \sim-5^{\circ} \mathrm{C}\right)$ is important to determine the ice crystal structure in frozen food. Although this concept is convenient to explain the relationship between the ice structure and the operating conditions in the freezing process, this cannot describe the ice structure quantitatively. On the contrary, the present result shows that the moving speed of the freezing front, $U$, is the most important parameter, representing all the differences in operating conditions and physical properties of food to be frozen, to describe the ice structure in frozen food quantitatively. Mean ice crystal size was inversely proportional to $U$, and the slope of the correlation line was in the same order as the diffusion coefficient of water, suggesting an important role of the molecular diffusion in the ice crystal growth process. 
Table 1. Ice crystal size frozen under various conditions.

\begin{tabular}{cccccc}
\hline $\begin{array}{c}\text { Agar } \\
\text { conc. }(\%)\end{array}$ & $\begin{array}{c}\text { Moving speed of } \\
\text { freezing front } U \\
\left(10^{-2} \mathrm{~m} / \mathrm{hr}\right)\end{array}$ & $\begin{array}{c}\text { Correction } \\
\text { value }\end{array}$ & $\begin{array}{c}\text { Mean minor } \\
\text { axis } \\
\left(10^{-6} \mathrm{~m}\right)\end{array}$ & $\begin{array}{c}\text { Mean major } \\
\text { axis } \\
\left(10^{-6} \mathrm{~m}\right)\end{array}$ & $\begin{array}{c}\text { Geometric mean } \\
\left(10^{-6} \mathrm{~m}\right)\end{array}$ \\
\hline 1.5 & 0.533 & 1.123 & 168.5 & 388.7 & 255.9 \\
1.5 & 0.686 & 1.109 & 139.4 & 310.3 & 208 \\
1.5 & 0.703 & 1.461 & 212.4 & 434.8 & 303.9 \\
1.5 & 0.899 & 1.102 & 145.2 & 299.6 & 208.6 \\
1.5 & 1.127 & 1.140 & 142.7 & 281.2 & 200.3 \\
1.5 & 1.499 & 1.116 & 127.7 & 273.7 & 187 \\
1.5 & 3.636 & 1.150 & 100.1 & 194 & 139.4 \\
1.5 & 7.843 & 1.140 & 61.9 & 128.2 & 89.1 \\
3 & 0.725 & 1.130 & 193.6 & 372.4 & 268.5 \\
3 & 0.82 & 1.130 & 152.8 & 345.1 & 229.6 \\
3 & 1.189 & 1.130 & 175.3 & 336.3 & 242.8 \\
3 & 1.55 & 1.130 & 179.6 & 347.5 & 249.8 \\
3 & 1.613 & 1.116 & 11.9 & 201.2 & 150 \\
3 & 2.174 & 1.130 & 145.5 & 284.8 & 203.6 \\
3 & 3.344 & 1.130 & 114.9 & 214 & 156.8 \\
3 & 5.102 & 1.111 & 101.2 & 184.4 & 136.6 \\
3 & 5.155 & 1.130 & 86.9 & 196.8 & 130.8 \\
5 & 1.139 & 1.130 & 163.5 & 315.3 & 227 \\
5 & 1.835 & 1.121 & 125.7 & 250.3 & 177 \\
5 & 4.608 & 1.130 & 111 & 228 & 159.1 \\
5 & 4.673 & 1.097 & 115.1 & 219.7 & 159 \\
\hline
\end{tabular}

Table 2. Comparison of $\alpha$ in Eq. (1) and $U d_{\mathrm{P}}$ in the literature.

\begin{tabular}{llc}
\hline Frozen sample & \multicolumn{1}{c}{$\begin{array}{c}\alpha \text { or } U d_{\mathrm{p}} \\
\left(10^{-9} \mathrm{~m}^{2} / \mathrm{s}\right)\end{array}$} \\
\hline Agar gel $(1.5 \%)$ & & 0.261 \\
Agar gel $(3.0 \%)$ & & 0.286 \\
Agar gel $(5.0 \%)$ & & 0.273 \\
Agar gel $(3.0 \%)^{* 1}$ & & 0.271 \\
Soy protein curd $(6.2 \%)^{* 2}$ & & 0.36 \\
Soy protein curd $(6.2 \%)^{* 3}$ & With ultrasound & 0.365 \\
Soy protein curd $(6.2 \%)^{* 3}$ & With Agl added & 0.2 \\
Soy protein curd $(6.2 \%)^{* 3}$ & With $\mathrm{CaCO}_{3}$ added & 0.365 \\
Egg albumin $(8.0 \%)^{* 3}$ & & 0.292 \\
\hline
\end{tabular}

*1: Bae et al. (1993)

*2: Miyawaki et al. (1992)

$*^{3}$ : Miyawaki (1995)

\section{References}

Bae, S.K., Miyawaki, O. and Yano, T. (1993). Ice structure size in frozen agar gels analyzed by mercury porosimetry. Biosci. Biotechnol. Biochem., 57, 1624-1627.

Bevilacqua, A., Zaritzky, N.E. and Calvelo, A. (1979). Histological measurements of ice in frozen beef. J. Food Technol., 14, 237-251.

Bolliger, S., Closs, C., Zeng, Y. and Windhab, E. (1999). In-line use of near infrared spectroscopy to measure structure parameters of frozen model sorbet. J. Food Eng., 38, 455-467.

Bomben, J.L. and King, C.J. (1982). Heat and mass transport in the freezing of apple tissue. J. Food Technol., 17, 615-632.

Chen, Y.L. and Pan, B.S. (1997). Morphological changes in tilapia muscle following freezing by airblast and liquid nitrogen methods. Int. J. Food Sci. Technol., 32, 159-168.

Chevalier, D., Le Bail, A. and Ghoul, M. (2000). Freezing and ice crystals formed in a cylindrical food model: part I Freezing at atmospheric pressure. J. Food Eng., 46, 277-285.

Evans, J., Adler, J., Mitchell, J., Blanshard, J. and Rodger, G. (1996). Use of confocal laser scanning microscope in conjunction with a conduction heat transfer stage in order to observe dynamically the freeze-thaw cycle in an autofluorescent substance and to measure ice crystal size in situ. Cryobiology, 33, 27-33.

Kerr, W.L., Kauten, R.J., McCarthy, M.J. and Reid, D.S. (1998). Monitoring the formation of ice during food freezing by magnetic resonance imaging. Lebensm. Wiss. Technol, 31, 215-220.

Miyawaki, O. (1995). Analysis of freeze-denaturation of soy protein and its control. Rep. Soy. Protein Res. Comm., 16, 100-103 (in Japanese).

Miyawaki, O. (2001). Analysis and control of ice crystal structure in frozen food and their application to food processing. Food Sci. Technol. Res., 7, 1-7.

Miyawaki, O., Abe, T. and Yano, T. (1989). A numerical model to describe freezing of foods when supercooling occurs. J. Food Eng., 9, 143-151.

Miyawaki, O., Abe, T. and Yano, T. (1992). Freezing and ice structure formed in protein gels. Biosci. Biotechnol. Biochem., 56 953-957.

Ngapo, T.M., Babare, I.H., Reynolds, J. and Mawson, R.F. (1999). Freezing rate and frozen storage effects on the ultrastructure of samples of pork. Meat Sci., 53, 159-168.

Reid, D.S. (1980). Cryomicroscope studies of the freezing process in tissue and in model systems. Int. J. Refrig., 3, 226-228.

Regand, A. and Goff, H.D. (2002). Effect of biopolymers on structure and ice recrystallization in dynamically frozen ice cream model systems. J. Dairy Sci., 85, 2722-2732.

Regand, A. and Goff, H.D. (2003). Structure and ice recrystallization in frozen stabilized ice cream model systems. Food Hydrocolloids, 17, 95-102.

Rohatgi, P.K. and Adams, C.M. (1967a). Effect of freezing rates on dendritic solidification of ice from aqueous solutions. Trans. Metallurg. Soc. AIME., 239, 1729-1736.

Rohatgi, P.K. and Adams, C.M. (1967b). Dendritic solidification of aluminum-copper alloys. Trans. Metallurg. Soc. AIME., 239, $1737-1746$.

Russell, A.B., Cheney, P.E. and Wantling, S.D. (1999). Influence of freezing conditions on ice crystallization in ice cream. J. Food Eng., 39, 179-191.

Tiller, W.A. and Rutter, J.W. (1956). The effect of growth conditions upon the solidification of a binary alloy. Can. J. Phys., 34, 96-121.

Trgo, C., Koxholt, M. and Kessler, H.G. (1999). Effect of freezing point and texture regulating parameters on the initial ice crystal growth in ice cream. J. Dairy Sci., 82, 460-465.

Woinet, B., Andrieu, J. and Laurent, M. (1998a). Experimental and theoretical study of model food freezing. Part I. Heat transfer modelling. J. Food Eng., 35, 381-393.

Woinet, B., Andrieu, J., Laurent, M. and Min, S.G. (1998b). Experimental and theoretical study of model food freezing. Part II. Characterization and modeling of the ice crystal size. J. Food Eng., 35, 395-407. 